\title{
MAXIMAL WEDGES OF SUBHARMONIC FUNCTIONS
}

\author{
BY L. L. HELMS
}

\author{
Communicated by M. M. Day, May 21, 1963
}

The application of the Perron-Wiener method of solving the Dirichlet problem for a diffusion operator, using only subharmonic functions in the domain of the operator, may fail since the domain of the operator may be too small. This leads to the consideration of extensions of the operator to enlarge the class of subharmonic functions. If maximal classes of subharmonic functions are used in the Perron-Wiener method, then it is possible to show that the Dirichlet problem for certain regular sets is solvable for a continuous boundary function if and only if the upper and lower Dirichlet solutions are continuous functions. With one exception, proofs will be omitted since details will appear in a forthcoming paper [1].

Let $C(X)$ be the usual Banach space of real bounded continuous functions on a separable locally compact metric space $X . P$ will denote a class of "subharmonic" functions in $C(\bar{W})$, where $W$ is an open subset of $X$ with compact closure $\bar{W}$ and nonempty boundary $W^{\prime}$, with the following properties: (i) $P$ contains the constant functions; (ii) $P-P$ is dense in $C(\bar{W})$; (iii) $P$ is a wedge in $C(\bar{W})$; and (iv) if $U$ is a nonempty open subset of $W$ with $\vec{U} \subset W$ and nonempty boundary $U^{\prime}$ and $f \in P$, then $f(\eta) \leqq \sup _{U^{\prime}} f$ for all $\eta \in \bar{U}$. A nonempty open set $U$ with $\bar{U} \subset W$ and nonempty boundary $U^{\prime}$ is called a regular set if $U^{\prime}=\nabla_{P} U$ where $\nabla_{P} U$ is the Choquet boundary of $U$ relative to $P$ (see [3] for the definition and existence of the Choquet boundary). It is assumed that the regular sets form a basis for the relative topology of $W$.

A wedge $R \subset C(\bar{W})$ is said to be compatible with $P$ if $R \supset P$ and $f \in R, U$ regular, $\eta \in \bar{U}$ implies $f(\eta) \leqq \sup _{U}, f$. The class of wedges compatible with $P$ can be partially ordered by set inclusion and contains a maximal element $Q$ by Zorn's lemma. Just as in [2], it can be shown that for each $\eta \in \bar{U}$, where $U$ is regular, there is a unit measure $\mu$ with support $S(\mu) \subset U^{\prime}$ such that $f(\eta) \leqq \int f d \mu$ for all $f \in Q . M(\eta, U, Q)$ will denote the set of all such $\mu$. The maximal wedge $Q$ possesses properties not possessed by $P$ in general. Not only is it true that $Q$ is closed under the operation of taking the maximum of two elements of $Q$, but it is also true that $Q$ is closed under an operation of taking the partial maximum of two elements of $Q$; i.e., if $f, g \in Q$ and there are open sets $U, V$ with $\vec{U} \subset V \subset W, f \geqq g$ on $U, f \leqq g$ on $V-U$, then the function which is equal to $f$ on $U$ and equal to $g$ on $\bar{W}-U$ is an 
element of $Q$. In order to prove these statements, a class $Q_{1}$ of functions which are locally subharmonic relative to the measures in $M(\eta, U, Q)$ is defined as follows. $Q_{1}$ consists of functions $f \in C(\bar{W})$ with the property that for each $\eta \in W$ and all sufficiently small regular neighborhoods $U$ of $\eta, f(\eta) \leqq \int f d \mu$ for all $\mu \in M(\eta, U, Q)$. $Q_{1}$ obviously contains $Q$; in fact, $Q_{1}=Q$. To show that $Q_{1}=Q$, it is only necessary to show that $Q_{1}$ is compatible with $P$. The only real problem here is that of showing that elements of $Q_{1}$ satisfy the maximum principle for regular sets knowing that they satisfy a local maximum principle. Since the definition of a regular set is weaker than that used in [1], a proof of this statement will be given. All of the results of [1] are valid if this weaker definition is used.

LEMma 1. If $f \in Q_{1}, U$ regular, and $\eta \in \bar{U}$, then $f(\eta) \leqq \sup _{U^{\prime}} f$.

Proof. Suppose there is an $\eta \in U$ such that $f(\eta)>\sup _{U}, f$. It can be assumed that $f(\eta)=\sup _{\bar{U}} f$. Let $E=\{\sigma: \sigma \in \vec{U}, f(\sigma)=f(\eta)\} \subset U$. $E$ is nonempty and compact. Consider $\nabla_{Q} E$, the Choquet boundary of $E$ relative to $Q \mid E$ (where $Q \mid E$ denotes restriction of elements of $Q$ to $E$ ). Let $\sigma_{0}$ be a point of $\nabla_{Q} E$. By definition of the Choquet boundary, $g\left(\sigma_{0}\right) \leqq \int g d \mu$ for all $g \in Q \mid E, S(\mu) \subset E \Rightarrow S(\mu)=\left\{\sigma_{0}\right\}$. Since $f$ is locally subharmonic, there is a small regular neighborhood $V_{\sigma_{0}}$ of $\sigma_{0}$ such that $\bar{V}_{\sigma_{0}} \subset U$ and $f\left(\sigma_{0}\right) \leqq \int f d \mu$ for all $\mu \in M\left(\sigma_{0}, V_{\sigma_{0}}, Q\right)$. Consider any such $\mu$. Suppose $S(\mu) \subset E$. Since $\sigma_{0} \in \nabla_{Q} E, S(\mu)=\left\{\sigma_{0}\right\}$ and a contra-

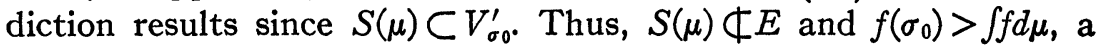
contradiction.

Using the maximality of $Q$, the following lemma is proved by considering the wedge spanned by $Q$ and an element not in $Q$.

Lemma 2. If $f \in C(\bar{W})$ but $f \in Q$, then there is an $\eta \in W$ and arbitrarily small regular neighborhoods $V$ of $\eta$ such that $f(\eta)>\int f d \mu$ for all $\mu \in M(\eta, V, Q)$.

If $U$ is any regular set and $x \in C\left(U^{\prime}\right)$, the upper and lower Dirichlet solutions are defined as $\mathfrak{u}^{U}(x)=\inf \left\{f|\vec{U}: f| U^{\prime} \geqq x, f \in-Q\right\}$ and $\mathfrak{R}^{U}(x)=\sup \left\{f|\vec{U}: f| U^{\prime} \leqq x, f \in Q\right\}$, respectively. The Dirichlet problem is discussed relative to regular sets only. It is possible to show that the upper and lower solutions satisfy an averaging principle.

THEOREM 3. Let $U$ and $V$ be regular sets with $V \subset U$ and let $x \in C\left(U^{\prime}\right)$. Then for $\eta \in \bar{V}, \mathbb{R}^{U}(x)(\eta)=\int \mathfrak{R}^{U}(x)(\sigma) p(\eta, V, d \sigma)$ for some $p(\eta, V, \cdot)$ $\in M(\eta, V, Q)$.

The proof of the following theorem is based upon the fact that a convex linear combination of $\mathfrak{R}^{U}(x)$ and $\mathfrak{H}^{U}(x)$, if continuous and 
$x=f \mid U^{\prime}$ for some $f \in Q$, cannot be an element of $Q$ if $\mathfrak{g}^{v}(x)$ and $\mathfrak{u}^{U}(x)$ are not equal.

THEOREM 4. If $U$ is a regular set, $x \in C\left(U^{\prime}\right)$, and $\mathfrak{R}^{U}(x)$ and $\mathfrak{u}^{U}(x)$ are continuous on $\bar{U}$, then $\mathfrak{R}^{U}(x)=\mathfrak{H}^{\mathcal{U}}(x)$.

The essential problem in showing that the Perron-Wiener method solves the Dirichlet problem for regular sets is that of showing that the upper and lower solutions are continuous. If $X$ is the set of real numbers and the class of regular sets is such that each point of $W$ is both the supremum of a regular set and infimum of a regular set, then it is possible to show that the upper and lower solutions are always continuous. A unified treatment of the one-dimensional case, without regard to regular or nonregular points for the operator, will be discussed in another forthcoming paper.

\section{REFERENCES}

1. L. Helms, The Perron-Wiener method for diffusion operators, Indiana J. Math. Mech. (to appear).

2. - A representation of the infinitesimal generator of a diffusion process, Bull. Amer. Math. Soc. 67 (1961), 479-482.

3. H. Bauer, Silovscher Rand und Dirichletsches Problem, Ann. Inst. Fourier (Grenoble) 11 (1961), 89-136.

UNIVERSITY OF ILLINOIS 